\title{
Population-based study of lung function and incidence of heart failure hospitalisations
}

\author{
Gunnar Engström, Olle Melander, Bo Hedblad
}

Department of Clinical Sciences, Lund University, Malmö University Hospital, Sweden

\section{Correspondence to} Professor Gunnar Engström, Cardiovascular Epidemiology Research Group, UMAS, CRC, 20502 Malmö, Sweden; Gunnar.Engstrom@med.lu.se

Received 17 January 2010 Accepted 19 April 2010

\section{ABSTRACT}

Background Reduced forced expiratory volume ( $\left.\mathrm{FEV}_{1}\right)$ and forced vital capacity (FVC) are risk factors for myocardial infarction (MI) and stroke. However, the relationship with incidence of heart failure (HF) is incompletely known. This population-based study explored whether reduced $\mathrm{FEV}_{1}$ or FVC is associated with the incidence of hospitalisation for $\mathrm{HF}$

Methods 20998 men (mean age 43 years) with no history of Ml or stroke were examined with spirometry in 1974-84. The incidence of hospitalisation due to HF was studied over a mean follow-up of 23 years in relation to age- and height-adjusted $\mathrm{FEV}_{1}$ and FVC.

Results 725 incident HF hospitalisations occurred during the follow-up period, 503 of them without a previous or concurrent diagnosis of Ml. In non-smokers the risk factor-adjusted hazard ratio (HR) for HF hospitalisation was 1.25 (95\% Cl 1.11 to 1.40$)$ and 1.26 (95\% Cl 1.13 to 1.42), respectively, per $1 \mathrm{SD}$ lower $\mathrm{FEV}_{1}$ and FVC. The corresponding risk factor-adjusted HRs in smokers were $1.32(95 \% \mathrm{Cl} 1.21$ to 1.45$)$ and 1.22 (95\% Cl 1.11 to 1.33), respectively, for FEV ${ }_{1}$ and FVC. This relationship was consistent in men with and without hypertension, in men above and below the median age of 44 years and for $\mathrm{HF}$ events without previous or concurrent Ml.

Exclusion of cases with a diagnosis of chronic obstructive pulmonary disease during the follow-up period did not substantially change the results.

Conclusion In this long-term population-based study of men, moderately reduced $\mathrm{FEV}_{1}$ and FVC were associated with an increased incidence of hospitalisations due to HF.

\section{INTRODUCTION}

Reduced lung function, measured by forced expiratory volume in $1 \mathrm{~s}\left(\mathrm{FEV}_{1}\right)$ or forced vital capacity (FVC), is a risk factor for cardiovascular disease (CVD). Low $\mathrm{FEV}_{1}$ and FVC within the normal range have been associated with the incidence of myocardial infarction (MI), stroke and asymptomatic atherosclerosis in the carotid and leg arteries. ${ }^{1-6}$ However, whether low $\mathrm{FEV}_{1}$ or $\mathrm{FVC}$ is similarly associated with the incidence of heart failure (HF) is unclear.

HF is a multifactorial disorder with several systemic manifestations which could be the result of different disease processes that reduce cardiac function. ${ }^{7-9}$ Reduced lung function is one of the systemic effects, and it has been known for many years that HF results in reduced lung volumes. ${ }^{10-12}$ However, the temporal and causal relationships between lung function and HF are unclear. In a population-based study of elderly people aged $>65$ years, low $\mathrm{FEV}_{1}$ was associated with an increased incidence of HF independently of other major risk factors. ${ }^{13}$ Similar relationships were reported from the Framingham study ${ }^{10}$ but not in the study of 'Men born in 1913'. ${ }^{14}$ It is largely unknown whether moderately reduced $\mathrm{FEV}_{1}$ or FVC in apparently healthy young or middle-aged subjects could be a risk factor for developing HF.

The Malmö Preventive Project includes approximately 21000 apparently healthy men with information on lung function and other cardiovascular risk factors. The cohort was examined at a mean age of 43 years-that is, at an age when HF is very unusual in men from the general population. The purpose of this follow-up was to explore whether $\mathrm{FEV}_{1}$ and FVC are associated with the incidence of hospitalisations due to HF.

\section{METHODS}

Between 1974 and 1984, 22444 men participated in a screening programme for detection of individuals with a high risk for CVD. ${ }^{15}$ Complete birth cohorts from the city of Malmö were invited to a health examination which included blood tests and a comprehensive questionnaire. The participation rate was $71 \%$. It has been reported that cardiovascular mortality was higher and that the incidence of non-fatal MI was lower in non-participants. ${ }^{15}$

Of the 22444 men, information about $\mathrm{FEV}_{1}$ and FVC was available in 21185 men. Subjects with missing information about blood pressure $(n=13)$, blood lipids $(\mathrm{n}=54)$ and body mass index (BMI) $(n=2)$ were excluded. To reduce the possibility that pre-existing heart disease reduced the lung volumes, we also excluded men with a history of MI or stroke $(n=114)$ and subjects with a hospital diagnosis of HF before the baseline examination $(n=5)$. A total of 20998 men remained after the exclusions. The mean (SD) age was 43.4 (6.6) years (range 27-61).

\section{Baseline examinations}

Subjects were categorised into smokers and nonsmokers. The smokers were categorised into consumers of up to 9 cigarettes per day, 10-19 cigarettes per day and a daily consumption of $\geq 20$ cigarettes.

BMI was calculated as weight/height ${ }^{2}\left(\mathrm{~kg} / \mathrm{m}^{2}\right)$.

Blood pressure $(\mathrm{mm} \mathrm{Hg})$ was measured twice in the right arm after $10 \mathrm{~min}$ of rest. The average of two measurements was used. A sphygmomanometer and a rubber cuff of appropriate size were used. Use of antihypertensive medication was assessed in a questionnaire. Hypertension was defined as systolic blood pressure $\geq 140 \mathrm{~mm} \mathrm{Hg}$ and/or diastolic blood pressure $\geq 90 \mathrm{~mm} \mathrm{Hg}$ and/or pharmacological treatment for hypertension.

Physical inactivity in spare time was assessed using the question: 'Are you mostly engaged in 
sedentary activities in spare time, for example, watching TV, reading, going to the movies?'. ${ }^{16}$ Subjects who confirmed a doctor's diagnosis of angina pectoris or who used nitrates were considered to have angina pectoris. ${ }^{16}$

Blood samples were taken after an overnight fast. Leucocyte counts were analysed using an automatic counter according to the standard methods at the hospital laboratory. Serum cholesterol, triglycerides and blood glucose were similarly analysed with standard methods at the hospital laboratory. Men with fasting whole blood glucose $\geq 6.1 \mathrm{mmol} / \mathrm{l}$ and those who reported treatment for diabetes were considered diabetic. Information on diabetes was missing for $74(0.4 \%)$ individuals. This group was coded in a separate category to keep them in the multivariate analysis.

High alcohol consumption was assessed by means of the modified shortened version of the Michigan Alcoholism Screening Test. ${ }^{17}$ Men with more than two (of nine) affirmative answers were considered to have high alcohol consumption. ${ }^{16}$

FVC and $\mathrm{FEV}_{1}$ were measured using a Spirotron apparatus (Drägerwerk AG, Lübeck, Germany) with the subjects in a standing position without noseclips. Specially trained nurses performed the tests. One acceptable manoeuvre with respect to the subject's performance and cooperation was required. The volumes were standardised for age and height using equations derived from linear regressions of 3467 male never smokers in the present cohort. $^{18} 19$ The following equations were used:

Predicted $\mathrm{FEV}_{1}(\mathrm{l})=4.422 \times$ height $(\mathrm{m})-0.038 \times$ age -2.48

Predicted FVC $(\mathrm{l})=6.58 \times$ height $(\mathrm{m})-0.033 \times$ age -5.54

Predicted $\mathrm{FEV}_{1} / \mathrm{FVC}(\%)=1.156-0.1556 \times$ height $(\mathrm{m})-$

$0.002025 \times$ age

Age and height were essentially unrelated to the lung volumes after adjustments. FEV 1 and FVC are expressed as percentage of the predicted values.

\section{Occupation and marital status}

Information on marital status and occupation was retrieved by data linkage with national census registers. ${ }^{20}$ High and medium level non-manual workers and self-employed men were categorised as 'high' occupational groups. Low level non-manual workers, manual workers and unspecified occupational groups (early retired men, students, unemployed) were categorised as 'low' occupational groups. The subjects were further categorised into single (divorced, widowers or unmarried) men or married (married or cohabiting) men. ${ }^{20}$ Information on occupation level and marital status was missing in $181(0.9 \%)$ and $56(0.3 \%)$ of the men, respectively. These groups were coded into separate categories to keep them in the multivariate analysis.

\section{Follow-up}

In the primary analysis, all men were followed from the baseline examination until first hospitalisation due to HF, death, emigration from Sweden or 31 December 2004, whichever came first. Subjects with a hospital discharge diagnosis of HF (codes 427.00, 427.10, 428.99, International Classification of Diseases, ICD-8), 428 (ICD-9) and I50 or I11.0 (ICD-10)) were considered to have $\mathrm{HF}$ if the diagnosis was listed as the primary diagnosis. In a secondary analysis, subjects with non-fatal MI were followed until the day of the infarction and censored thereafter. A non-fatal MI was defined as code 410 or I21 according to ICD-9 or ICD-10.

Since low lung function is a major risk factor for developing chronic obstructive pulmonary disease (COPD), and COPD hypothetically could increase the probability of being hospitalised and having a diagnosis of HF, an additional analysis was performed in which subjects who developed COPD were followed until the first diagnosis of COPD, listed as one of the first three diagnoses, ${ }^{18}$ and censored after that.

The Swedish Hospital Discharge Register was used for case retrieval and the Swedish population register was used for information on emigration and mortality during the follow-up. A validation study has shown that a primary diagnosis of $\mathrm{HF}$ in the Swedish Hospital Discharge Register has a validity of $95 \%{ }^{21}$

\section{Statistical analysis}

One-way analysis of variance (for continuous variables) and logistic regression (for dichotomous variables) was used for cross-sectional analyses of the risk factor distribution over the quartiles of $\mathrm{FEV}_{1}$. The Kaplan-Meier test with log rank statistics was used to study the incidence of HF hospitalisations for quartiles of lung function. Cox proportional hazards regression was used to calculate risk factor-adjusted hazard rates (HR). Cardiovascular risk factors that were associated with $\mathrm{FEV}_{1}$ at the baseline examination were selected as covariates in the multivariate analysis. Age, cholesterol, triglycerides (log-normalised), systolic blood pressure and BMI were fitted as continuous variables. Smoking, antihypertensive treatment, high alcohol consumption and physical inactivity were fitted as dichotomous variables. Three categories were used for low occupation level, married/cohabiting and diabetes (yes, no, missing). The fit of the proportional hazards model was checked visually by plotting the incidence rates over time and by entering time-dependent variables into the model. The covariate-adjusted Cox analysis was performed separately in smokers and non-smokers. Finally, the relationship between lung function and HF was explored for cases without a history of MI during the follow-up period and after censoring men who were diagnosed with COPD before the onset of HF.

\section{RESULTS}

\section{Study cohort}

In terms of unadjusted volumes, mean $\pm \mathrm{SD} \mathrm{FEV}_{1}$ was $3.7 \pm 0.751$ and $3.4 \pm 0.761$, respectively, in non-smokers and smokers. FVC was $4.7 \pm 0.891$ and $4.4 \pm 0.901$, respectively. Expressed as a percentage of the predicted values, mean $\pm \mathrm{SD} \mathrm{FEV}_{1}$ was $99 \pm 17 \%$ in non-smokers and $91 \pm 17 \%$ in smokers, and mean \pm SD FVC was $99 \pm 16 \%$ in non-smokers and $94 \pm 16 \%$ in smokers. $\mathrm{FEV}_{1}$ was $94 \pm 18 \%, 91 \pm 17 \%$ and $88 \pm 17 \%$, respectively, in smokers with consumption of $<10,10-19$ and $\geq 20$ cigarettes per day.

The baseline characteristics of the study population are shown in table 1 in relation to quartiles of $\mathrm{FEV}_{1}$. All major cardiovascular risk factors showed significant relationships with $\mathrm{FEV}_{1}$.

\section{Incidence of $\mathbf{H F}$ in relation to lung function}

During the mean follow-up period of $23.0 \pm 5.5$ years, 725 men were admitted to hospital with a primary diagnosis of HF. Of these, 222 had been admitted with a primary diagnosis of MI before the HF event. Low FEV 1 and low FVC were significantly associated with the incidence of HF. This relationship was consistent in smokers and non-smokers, also after adjustments for cardiovascular risk factors (figures 1 and 2 and table 2). $\mathrm{FEV}_{1}$ / FVC (\% predicted) in the lowest quartile was associated with HF hospitalisations in smokers. There was no relationship between $\mathrm{FEV}_{1} / \mathrm{FVC}$ and the incidence of $\mathrm{HF}$ in non-smokers.

A total of 503 cases had HF without MI before or concurrent with the diagnosis of HF. The relationship between low lung function and incidence of HF was even stronger for these cases (table 3). 
Table 1 Baseline characteristics of the study cohort in relation to quartiles of forced expiratory volume in $1 \mathrm{~s}\left(\mathrm{FEV}_{1}\right)$

\begin{tabular}{|c|c|c|c|c|c|c|}
\hline & 04 (high) & 03 & 02 & 01 (low) & $\mathbf{p}_{\text {trend }}$ & All men \\
\hline $\mathrm{N}$ & 5249 & 5250 & 5250 & 5249 & & 20998 \\
\hline $\mathrm{FEV}_{1}, \%$ predicted & $>106$ & $95-106$ & $84-95$ & $<84$ & & $95 \pm 18$ \\
\hline Age, years & $43.9 \pm 6.5$ & $43.0 \pm 6.6$ & $42.8 \pm 6.7$ & $44.1 \pm 6.7$ & 0.32 & $43.4 \pm 6.6$ \\
\hline Height, cm & $177 \pm 6.7$ & $177 \pm 6.7$ & $177 \pm 6.7$ & $177 \pm 6.9$ & 0.76 & $177 \pm 6.7$ \\
\hline Current smoking, $\%$ & 36.0 & 43.9 & 53.8 & 64.4 & $<0.001$ & 49.5 \\
\hline$>20$ cigs $/$ day, $\%$ of smokers & 19.4 & 23.6 & 28.5 & 33.1 & $<0.001$ & 27.3 \\
\hline Diabetes, $\%$ & 3.9 & 4.6 & 4.2 & 6.7 & $<0.001$ & 4.8 \\
\hline Systolic BP, mm Hg & $127 \pm 14$ & $127 \pm 14$ & $126 \pm 15$ & $128 \pm 16$ & $<0.001$ & $127 \pm 15$ \\
\hline Diastolic BP, mm Hg & $85.0 \pm 9.1$ & $85.1 \pm 9.4$ & $85.1 \pm 9.6$ & $86.2 \pm 10$ & $<0.001$ & $85.4 \pm 10$ \\
\hline Antihypertensive treatment, \% & 3.1 & 3.5 & 3.6 & 4.9 & $<0.001$ & 3.8 \\
\hline Cholesterol, $\mathrm{mmol} / \mathrm{l}$ & $5.5 \pm 1.0$ & $5.6 \pm 1.0$ & $5.6 \pm 1.1$ & $5.7 \pm 1.1$ & $<0.001$ & $5.6 \pm 1.1$ \\
\hline Triglycerides, mmol/l† & 1.21 & 1.27 & 1.33 & 1.42 & $<0.001$ & 1.31 \\
\hline $\mathrm{BMI}, \mathrm{kg} / \mathrm{m}^{2}$ & $24.5 \pm 3.0$ & $24.6 \pm 3.2$ & $24.6 \pm 3.3$ & $24.9 \pm 3.7$ & $<0.001$ & $24.6 \pm 3.3$ \\
\hline Physical inactivity, \% & 46.1 & 50.7 & 54.4 & 59.0 & $<0.001$ & 52.6 \\
\hline Low occupation level, \% & 56.3 & 57.7 & 60.8 & 66.2 & $<0.001$ & 60.2 \\
\hline Married, $\%$ & 70.6 & 72.0 & 69.8 & 66.4 & $<0.001$ & 69.7 \\
\hline High alcohol consumption, \% & 15.6 & 16.8 & 18.7 & 20.7 & $<0.001$ & 18.0 \\
\hline Angina pectoris, $\%$ & 1.0 & 1.0 & 1.0 & 2.0 & $<0.001$ & 1.3 \\
\hline Leucocytes, $10^{9} / I^{*}$ & $5.7 \pm 1.8$ & $5.9 \pm 1.9$ & $6.2 \pm 2.0$ & $6.5 \pm 2.1$ & $<0.001$ & $6.1 \pm 2.0$ \\
\hline
\end{tabular}

*Leucocyte measurements were available in a subcohort of 15929 men.

†Triglycerides are shown as geometric means.

BMI, body mass index.

The death rates were also higher in subjects with low lung function. In non-smokers (1591 deaths), the death rates (per 1000 person-years) were 5.8, 5.7, 5.7 and 9.1, respectively, for the 4 th, 3rd, 2nd and 1 st quartiles of $\mathrm{FEV}_{1}$. In smokers (2909 deaths) the death rates were 9.3, 10.6, 11.6 and 16.2, respectively, in the quartiles of $\mathrm{FEV}_{1}$.

An additional analysis was performed in which patients were also censored if they had COPD listed as a hospital diagnosis during the follow-up period. A total of 263 incident cases of HF remained among the non-smokers and 371 cases remained among the smokers. After adjustments for risk factors, the relationship between $\mathrm{FEV}_{1}$ and the incidence of $\mathrm{HF}$ was 1.16 per 1SD (95\% CI 1.02 to 1.30) in non-smokers and 1.18 (95\% CI 1.06 to 1.31$)$ in smokers. The HRs for FVC were 1.19 per $1 \mathrm{SD}(95 \%$ CI 1.05 to 1.34$)$ in non-smokers and 1.14 (95\% CI 1.03 to 1.26$)$ in smokers. $\mathrm{FEV}_{1} / \mathrm{FVC}$ was not associated with $\mathrm{HF}$ in this analysis

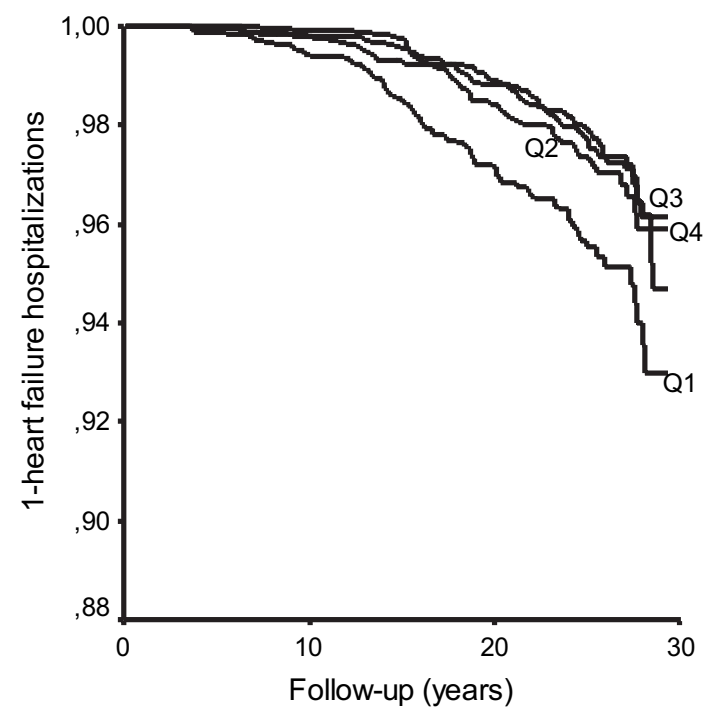

Figure 1 Incidence of hospitalisations for heart failure in non-smokers in relation to quartiles of forced expiratory volume in $1 \mathrm{~s}$. in non-smokers (HR per 1SD 1.00; 95\% CI 0.88 to 1.14 ) or smokers (HR 1.07; 95\% CI 0.97 to 1.17 ).

\section{Analyses stratified by hypertension and age}

The relationships between $\mathrm{FEV}_{1}$ and FVC, respectively, and the incidence of HF hospitalisations are shown in table 3, stratified by smoking, age and hypertension. The relationships with the incidence of HF were consistent in these subgroups.

The relationship between low lung function and the incidence of HF seemed to be even stronger for cases of HF without previous MI (table 3 ).

\section{Adjustments for leucocyte concentrations}

Information about leucocyte concentrations was available in a subgroup of 15929 men, 586 of whom were admitted to hospital with HF during the follow-up period. This subgroup

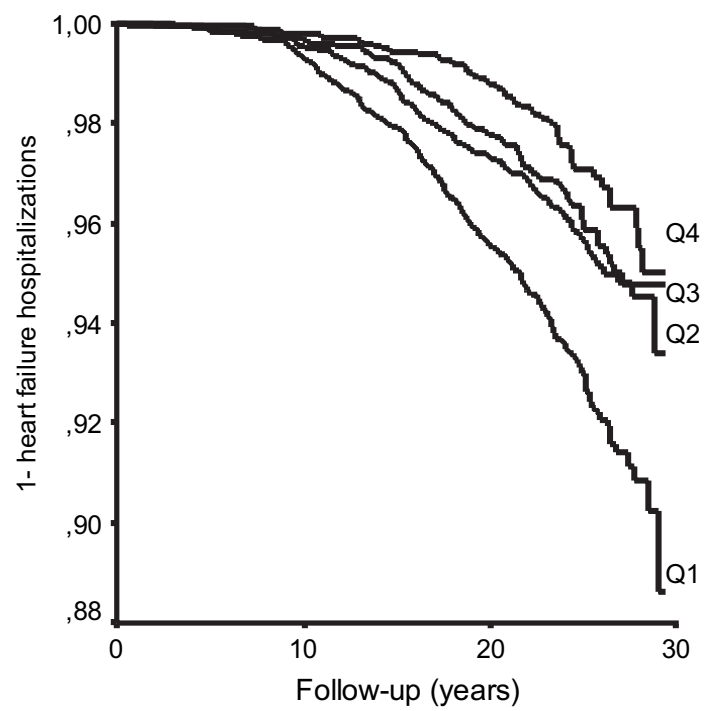

Figure 2 Incidence of hospitalisations for heart failure in smokers in relation to quartiles of forced expiratory volume in $1 \mathrm{~s}$. 
Table 2 Incidence of hospitalisations for HF in relation to measures of lung function in non-smokers and smokers

\begin{tabular}{|c|c|c|c|c|c|c|}
\hline & 04 (high) & 03 & 02 & 01 (low) & $\mathbf{p}_{\text {trend }}$ & HR per 1SD* \\
\hline \multicolumn{7}{|l|}{ Non-smokers } \\
\hline \multicolumn{7}{|l|}{$\mathrm{FEV}_{1}$} \\
\hline $\mathrm{N}$ & 3359 & 2943 & 2428 & 1870 & & \\
\hline Age-adjusted & 1.00 & 1.03 (0.74 to 1.44$)$ & $1.29(0.93$ to 1.80$)$ & 1.95 (1.42 to 2.66$)$ & $<0.001$ & \\
\hline Risk factors & 1.00 & 0.89 (0.64 to 1.25$)$ & 1.05 (0.75 to 1.47$)$ & 1.47 (1.07 to 2.02$)$ & 0.015 & 1.25 (1.11 to 1.40$)$ \\
\hline \multicolumn{7}{|l|}{ FVC } \\
\hline Age-adjusted & 1.00 & $1.22(0.87$ to 1.72$)$ & 1.22 (0.86 to 1.72 ) & 2.30 (1.68 to 3.15$)$ & $<0.001$ & \\
\hline Risk factors & 1.00 & $1.16(0.82$ to 1.63$)$ & 1.02 (0.71 to 1.45$)$ & 1.72 (1.24 to 2.37$)$ & 0.003 & $1.26(1.13$ to 1.42$)$ \\
\hline \multicolumn{7}{|l|}{$\mathrm{FEV}_{1} / \mathrm{FVC}$} \\
\hline $\mathrm{N}$ & 2909 & 2896 & 2652 & 2143 & & \\
\hline$H F(n)$ & 82 & 69 & 65 & 66 & & \\
\hline \multicolumn{7}{|l|}{$\mathrm{FEV}_{1}$} \\
\hline $\mathrm{N}$ & 1890 & 2307 & 2822 & 3379 & & \\
\hline $\mathrm{HF}(\mathrm{n})$ & 50 & 81 & 109 & 203 & & \\
\hline Age-adjusted & 1.00 & 1.46 (1.02 to 2.07$)$ & 1.61 (1.15 to 2.25$)$ & 2.41 (1.77 to 3.28 ) & $<0.001$ & \\
\hline Risk factors & 1.00 & 1.35 (0.95 to 1.93$)$ & 1.52 (1.09 to 2.13 ) & 2.05 (1.49 to 2.80$)$ & $<0.001$ & $1.32(1.21$ to 1.45$)$ \\
\hline \multicolumn{7}{|l|}{ FVC } \\
\hline $\mathrm{N}$ & 2119 & 2390 & 2696 & 3193 & & \\
\hline$H F(n)$ & 65 & 83 & 111 & 184 & & \\
\hline Age-adjusted & 1.00 & $1.19(0.86$ to 1.65$)$ & $1.47(1.09$ to 2.00$)$ & 1.91 (1.44 to 2.53 ) & $<0.001$ & \\
\hline Risk factors & 1.00 & $1.13(0.82$ to 1.57$)$ & 1.36 (1.00 to 1.85$)$ & 1.51 (1.13 to 2.02$)$ & 0.002 & $1.22(1.11$ to 1.33$)$ \\
\hline \multicolumn{7}{|l|}{$\mathrm{FEV}_{1} / \mathrm{FVC}$} \\
\hline $\mathrm{N}$ & 2340 & 2354 & 2598 & 3106 & & \\
\hline
\end{tabular}

Risk factors included systolic BP, antihypertensive treatment, cigarettes (smokers only), cholesterol, triglycerides, body mass index, physical inactivity, occupation level, diabetes, angina, marital status and high alcohol intake.

Median (interquartile limits) are $95 \%(84,106)$ for $\mathrm{FEV}_{1}, 97 \%(87,107)$ for $\mathrm{FVC}$ and $99 \%(92,105)$ for $\mathrm{FEV}_{1} / \mathrm{FVC}$.

*Hazard ratios (HR) per 1 SD lower FEV F $_{1}\left(18 \%\right.$ of predicted values), FVC ( $16 \%$ of predicted values) or $\mathrm{FEV}_{1} / \mathrm{FVC}(11 \%$ of predicted values).

$\mathrm{FEV}_{1}$, forced expiratory volume in $1 \mathrm{~s}$; $\mathrm{FVC}$, forced vital capacity; $\mathrm{HF}$, heart failure.

was somewhat older $(43.7 \pm 5.5$ vs $42.5 \pm 9.3$ years $)$ and more often smokers ( $51 \%$ vs $45 \%$ ) than those without information on leucocytes. The relationship between $\mathrm{FEV}_{1}$ and FVC, respectively, and the incidence of HF was only marginally reduced by adjustments for leucocytes, and the associations remained significant in smokers and non-smokers. For example, in nonsmokers the HR for 1SD lower FEV 1 was 1.25 (95\% CI 1.10 to 1.42) after adjustments for risk factors and 1.24 (95\% CI 1.09 to 1.42) after further adjustments for leucocytes; the corresponding HRs for FVC were 1.26 (95\% CI 1.11 to 1.44$)$ and 1.26 (95\% CI 1.10 to 1.44$)$, respectively.

\section{DISCUSSION}

Even though reduced $\mathrm{FEV}_{1}$ and FVC are well-known risk factors for CVD, the relationship between lung function and the incidence of HF is incompletely known. In this population-based study of men without a history of MI or stroke, reduced volumes of $\mathrm{FEV}_{1}$ or FVC were significantly associated with the incidence of HF requiring admission to hospital. This relationship was consistent in smokers and non-smokers, in men with and without hypertension and in men above and below the median age of 44 years. Pulmonary obstruction as measured by the $\mathrm{FEV}_{1} / \mathrm{FVC}$ ratio was associated with $\mathrm{HF}$ hospitalisations in smokers only.
The results are in accordance with a study of older subjects ${ }^{13}$ and the results from the Framingham study in which lung function was measured shortly before the HF diagnosis. ${ }^{10}$ Correlations between echocardiographic measures of left ventricular function and measures of airway obstruction were recently reported in a cross-sectional study. ${ }^{22}$ However, there was no relationship between lung function and the incidence of HF in the study 'Men Born in 1913' ${ }^{15}$ After adjustments for age and sex, COPD was not associated with echocardiographic findings in the Copenhagen Heart Study. ${ }^{23}$ The present results add to the current knowledge by demonstrating that reduced lung function is a very early risk factor which is predictive even in young age groups when very few people have cardiac failure, many years before the cases are hospitalised with HF. The present results persisted after adjustments for several risk factors including biological, socioeconomic and lifestyle factors, and the results could not be explained by relationships between lung function and the incidence of MI.

It has previously been shown that moderately reduced lung function is associated with the incidence of MI, stroke and subclinical atherosclerosis. ${ }^{1-6}$ However, the relationships with the incidence of HF are more complex and involve some methodological issues that need to be discussed. Patients with HF and those with COPD often show similar symptoms, and 
Table 3 Multivariate analysis of incidence of hospitalisations for $\mathrm{HF}$ in relation to $\mathrm{FEV}_{1}$ and $\mathrm{FVC}$ by age and hypertension status

\begin{tabular}{llll}
\hline & Cases/subjects (n/n) & FEV $_{\mathbf{1}}$ (per $\left.\mathbf{1 S D}\right)$ & FVC (per $1 \mathbf{S D})$ \\
\hline Non-smokers & & & \\
$<45$ years & $54 / 5467$ & $1.68(1.23$ to 2.30$)$ & $1.77(1.31$ to 2.39$)$ \\
$\geq 45$ years & $228 / 5133$ & $1.19(1.05$ to 1.35$)$ & $1.19(1.05$ to 1.35$)$ \\
Normal BP & $69 / 5569$ & $1.55(1.26$ to 1.92$)$ & $1.53(1.23$ to 1.90$)$ \\
Hypertension & $213 / 5031$ & $1.15(1.01$ to 1.32$)$ & $1.18(1.03$ to 1.35$)$ \\
HF, no Ml* & $207 / 10600$ & $1.30(1.14$ to 1.49$)$ & $1.33(1.17$ to 1.52$)$ \\
HF, after Ml $^{*}$ & $75 / 10600$ & $1.11(0.89$ to 1.39$)$ & $1.09(0.87$ to 1.37$)$
\end{tabular}

\section{Smokers}

$\begin{array}{llll}<45 \text { years } & 100 / 5220 & 1.34(1.09 \text { to } 1.66) & 1.22(0.99 \text { to } 1.51) \\ \geq 45 \text { years } & 343 / 5178 & 1.32(1.19 \text { to } 1.46) & 1.21(1.09 \text { to } 1.35) \\ \text { Normal BP } & 181 / 6532 & 1.36(1.18 \text { to } 1.58) & 1.27(1.10 \text { to } 1.47) \\ \text { Hypertension } & 262 / 3866 & 1.30(1.16 \text { to } 1.47) & 1.18(1.04 \text { to } 1.33) \\ \text { HF, no MI* } & 296 / 10398 & 1.38(1.24 \text { to } 1.54) & 1.22(1.09 \text { to } 1.37) \\ \text { HF, after Ml* } & 147 / 10398 & 1.21(1.03 \text { to } 1.42) & 1.21(1.03 \text { to } 1.41)\end{array}$

Presented as hazard ratios (per 1 SD lower FEV 1 or FVC) adjusted for systolic BP, antihypertensive treatment, cigarettes (smokers only), cholesterol, triglycerides, body mass index, physical inactivity, occupation level, diabetes, angina, marital status and high alcohol intake.

*Incidence of HF without previous myocardial infarction (no Ml) and with previous or concurrent Ml (after MI).

$\mathrm{FEV}_{1}$, forced expiratory volume in $1 \mathrm{~s}$; FVC, forced vital capacity; $\mathrm{HF}$, heart failure; $\mathrm{Ml}$, myocardial infarction.

misclassification of HF and lung disease is one potential cause of bias. $^{2425}$ Coexisting COPD could also increase the probability of hospitalisation for a patient with HF. However, since COPD is strongly linked to smoking, this potential misclassification bias would be much stronger in smokers than in non-smokers. A validation study using data from the Swedish Hospital Discharge Register showed that a diagnosis of HF had very high accuracy if it was listed as the primary diagnosis. ${ }^{21}$ To further reduce the risk of diagnostic misclassification and hospitalisation bias, we also performed an analysis in which men with a diagnosis of COPD were censored. $\mathrm{FEV}_{1} / \mathrm{FVC}$ was no longer associated with $\mathrm{HF}$ in smokers, but the relationships between $\mathrm{FEV}_{1}$, FVC and the incidence of HF were essentially the same. We therefore think it is unlikely that diagnostic misclassification could explain the results in this study.

Even though the relationship between reduced lung function and the incidence of CVD has been known for many years, the reason for this relationship is still unclear. Both $\mathrm{FEV}_{1}$ and FVC showed significant relationships with the incidence of HF in this study. This suggests that the results are related to lung volume or reduced compliance of the lung or chest wall, rather than to airway obstruction which would affect $\mathrm{FEV}_{1} / \mathrm{FVC}$ to a higher degree than FVC. Since the relationships were also clearly significant in young non-smokers (aged 27-44 years, mean 38 years), it is possible that the increased risk reflects traits which are established early in life or factors of genetic origin. Low birth weight has been associated with both increased cardiovascular risk and low $\mathrm{FEV}_{1}$ later in life ${ }^{26} 27$ and could be a possible link between $\mathrm{FEV}_{1}$ and CVD.

Ischaemic heart disease is a risk factor for $\mathrm{HF}$ and one question is whether this could explain the results. Men with a history of MI and stroke at baseline were excluded. The relationships were even stronger for HF cases without a previous or concurrent diagnosis of MI during the follow-up period. Hence, relationships between reduced lung function and clinical MI could not explain the results. However, many MIs are silent and it is possible that some incident cases of HF had a subclinical infarction.
Blood pressure is another major risk factor for HF. All analyses were adjusted for systolic blood pressure and antihypertensive treatment. We performed separate analyses of men with normal blood pressure, and low $\mathrm{FEV}_{1}$ or FVC was also associated with the incidence of HF in this group. It is unlikely that differences with respect to blood pressure at baseline confounded the relationship between lung function and the incidence of HF. However, some studies have reported that low FVC could be a risk factor for developing hypertension and diabetes. ${ }^{28-30}$ In that case, development of hypertension and diabetes during the follow-up period could be part of the causal chain between low lung function and HF. This possibility cannot be excluded. It has also been shown that reduced $\mathrm{FEV}_{1}$ is associated with increased 'beat-to-beat' variability of the systolic blood pressure, even within the normal range of $\mathrm{FEV}_{1} \cdot{ }^{31}$ Short-term variability in blood pressure is strongly related to the mechanical effects of breathing and variations in intrathoracic pressure and could therefore be related to lung function per se. ${ }^{32}$ Although blood pressure variability is a strong cardiovascular risk factor, ${ }^{33}$ the role of high frequent 'beat-to-beat' variations is unclear. Although speculative, it is possible that increased blood pressure variability could increase the risk of $\mathrm{HF}$ in men with low $\mathrm{FEV}_{1}$.

Increased cardiac size and increased pressure of the pulmonary circulation are two factors that reduce the lung volumes in patients with HF. One question is whether the results could be explained by reverse causation - that is, whether impaired lung function could be secondary to cardiac dysfunction. However, the men in this population study were rather young and HF in the absence of MI is very unusual at this age. The results persisted when the sample was restricted to men aged 44 years and younger. Only a few cases of HF occurred during the first 10 years of the follow-up period. The long time period between the baseline examination and the incidence of $\mathrm{HF}$ also reduces the possibility of reverse causation.

Recent studies have shown that systemic low-grade inflammation is associated with $\mathrm{HF}^{16} 34{ }^{35}$ Since low FEV 1 or FVC has been associated with raised plasma levels of various markers of inflammation, we performed a subgroup analysis of almost 16000 men with adjustments for the leucocyte concentration. A high leucocyte concentration has been associated with the incidence of $\mathrm{HF}$ in this cohort. ${ }^{16}$ The results were largely unchanged after adjustments for leucocytes, which suggests that inflammation has no major role in the relationship between lung function and HF.

Some limitations should be acknowledged. Only middle-aged men were studied, and it is unclear whether the results could be generalised to all age groups and to women. FEV 1 and FVC were measured several years before the currently used guidelines for standardisation of spirometry were published and the examinations did not meet the standards of the recent guidelines. If anything, low precision of the lung function measurements should reduce the association with the incidence of $\mathrm{HF}$. However, the validity is supported by previous studies from the present cohort which show that $\mathrm{FEV}_{1}$ and FVC are strongly linked to cardiovascular as well as pulmonary diseases. ${ }^{18} 1930$

All cases were admitted to hospital with a primary diagnosis of $\mathrm{HF}$, and it can be assumed that the cardiac disease was quite severe in most cases. We do not know to what extent lung function is associated with less severe forms of HF which are more likely to be treated in primary care. However, results from an echocardiographic follow-up of elderly subjects suggest that low $\mathrm{FEV}_{1}$ is also associated with the development of less severe forms of $\mathrm{HF}^{13}$ Another shortcoming is that we had limited 
information about the cases with regard to the type and cause of cardiac dysfunction.

In conclusion, this study of comparatively young men without a history of MI or stroke showed that moderately reduced volumes of $\mathrm{FEV}_{1}$ and $\mathrm{FVC}$ were associated with an increased incidence of HF requiring hospitalisation. This relationship was consistent in smokers and non-smokers, in men with and without hypertension and in men above and below the median age of 44 years.

Funding This study was supported by grants from the Swedish Heart and Lung Foundation, the Swedish Research Council, Lund University and Malmö University Hospital.

Competing interests GE is employed as a senior epidemiologist by AstraZeneca RGD.

Ethics approval This study was conducted with the approval of the regional ethics commitee, Lund University, Sweden. The health service authority of Malmö approved and funded the screening programme. All participants gave informed consent. The data linkage with hospitalisation registers was approved by the regional ethics committee.

Provenance and peer review Not commissioned; externally peer reviewed.

\section{REFERENCES}

1. Sin DD, Wu L, Man SF. The relationship between reduced lung function and cardiovascular mortality: a population-based study and a systematic review of the literature. Chest 2005:127:1952-9.

2. Johnston AK, Mannino DM, Hagan GW, et al. Relationship between lung function impairment and incidence or recurrence of cardiovascular events in a middle-aged cohort. Thorax 2008;63:599-605.

3. Hole DJ, Watt GCM, Davey-Smith G, et al. Impaired lung function and mortality risk in men and women: findings from the Renfrew and Paisley prospective population study. BMJ 1996:313:711-15.

4. Engström G, Hedblad B, Valind S, et al. Asymptomatic leg and carotid atherosclerosis in smokers is related to degree of ventilatory capacity: Iongitudinal and cross-sectional results from 'Men born in 1914', Sweden. Atherosclerosis 2001;155:237-43.

5. Zureik M, Kauffmann F, Touboul PJ, et al. Association between peak expiratory flow and the development of carotid atherosclerotic plaques. Arch Intern Med 2001;161:1669-76.

6. Lange $\mathbf{P}$, Nyboe J, Appleyard M, et al. Spirometric findings and mortality in neversmokers. J Clin Epidemiol 1990:43:867-73.

7. Cowie MR, Wood DA, Coats AJ, et al. Incidence and aetiology of heart failure: a population-based study. Eur Heart J 1999;20:421-8.

8. Butler J, Kalogeropoulos A, Georgiopoulou V; for the Health ABC Study. Incident heart failure prediction in the elderly: the Health $A B C$ heart failure score. Circ Heart Fail 2008:1:125-33.

9. Wilhelmsen L, Rosengren A, Eriksson $\mathrm{H}$, et al. Heart failure in the general population of men-morbidity, risk factors and prognosis. J Intern Med 2001;249:253-61.

10. Kannel WB, Seidman JM, Fercho W, et al. Vital capacity and congestive heart failure. The Framingham Study. Circulation 1974;49:1160-6.

11. Calhoun JA, Cullen GE, Harrison TR, et al. Studies in congestive heart failure: XIV. Orthopnea: its relation to ventilation, vital capacity, oxygen saturation and acid-base condition of arterial and jugular blood. J Clin Invest 1931;10:833-55.

12. Enright PL, Kronmal RA, Smith VE, et al. Reduced vital capacity in elderly persons with hypertension, coronary heart disease, or left ventricular hypertrophy. The Cardiovascular Health Study. Chest 1995;107:28-35.
13. Gottdiener JS, Arnold AM, Aurigemma GP, et al. Predictors of congestive heart failure in the elderly: the Cardiovascular Health Study. J Am Coll Cardiol 2000;35:1628-37

14. Eriksson H, Svärdsudd K, Larsson B, et al. Risk factors for heart failure in the genera population: the study of men born in 1913. Eur Heart $J$ 1989;10:647-56.

15. Berglund G, Nilsson P, Eriksson KF, et al. Long-term outcome of the Malmö preventive project: mortality and cardiovascular morbidity. J Intern Med 2000;247:19-29

16. Engström G, Melander 0, Hedblad B. Leukocyte count and incidence of hospitalizations due to heart failure. A population-based cohort study. Circ Heart Fail 2009;2:217-22.

17. Kristenson $\mathbf{H}$, Trell $\mathrm{E}$. Indicators of alcohol consumption: comparisons between a questionnaire (Mm-MAST), interviews and serum gamma-glutamyl transferase (GGT) in a health survey of middle-aged males. Br J Addict 1982;7:297-304.

18. Engström G, Segelstorm N, Ekberg-Aronsson M, et al. Plasma markers of inflammation and incidence of hospitalisations for COPD: results from a populationbased cohort study. Thorax 2009:64:211-15.

19. Ekberg-Aronsson M, Pehrsson K, Nilsson JA, et al. Mortality in GOLD stages of COPD and its dependence on symptoms of chronic bronchitis. Respir Res 2005;6:98

20. Hedblad $\mathbf{B}$, Jonsson $\mathrm{S}$, Nilsson $\mathrm{P}$, et al. Obesity and myocardial infarction: vulnerability related to occupational level and marital status. A 23-year follow-up of an urban male Swedish population. J Intern Med 2002;252:542-50.

21. Ingelsson $\mathbf{E}$, Arnlöv J, Sundström J, et al. The validity of a diagnosis of heart failure in a hospital discharge register. Eur J Heart Fail 2005; 7:787-91.

22. Barr RG, Bluemke DA, Ahmed FS, et al. Percent emphysema, airflow obstruction, and impaired left ventricular filling. N Engl J Med 2010;362:217-27.

23. Lange $\mathbf{P}$, Mogelvang R, Marott JL, et al. Cardiovascular morbidity in COPD: a study of the general population. COPD 2010:7:5-10.

24. Hawkins NM, Petrie MC, Jhund PS, et al. Heart failure and chronic obstructive pulmonary disease: diagnostic pitfalls and epidemiology. Eur J Heart Fail 2009;11:130-9.

25. Rutten FH, Cramer MJ, Lammers JW, et al. Heart failure and chronic obstructive pulmonary disease: an ignored combination? Eur J Heart Fail. 2006;8:706-11.

26. Barker DJ, Godfrey KM, Fall C, et al. Relation of birth weight and childhood respiratory infection to adult lung function and death from chronic obstructive airways disease. BMJ 1991;303:671-5.

27. Barker DJ, Osmond C, Forsén TJ, et al. Trajectories of growth among children who have coronary events as adults. N Engl J Med 2005;353:1802-9.

28. Sparrow D, Weiss ST, Vokonas PS, et al. Forced vital capacity and the risk of hypertension. The Normative Aging Study. Am J Epidemiol 1988;127:734-41.

29. Engström G, Wollmer $P$, Valind S, et al. Blood pressure increase between 55 and 68 years of age is inversely related to lung function: Iongitudinal results from the cohor study 'Men born in 1914'. J Hypertens 2001;19:1203-8.

30. Engström G, Hedblad B, Nilsson $P$, et al. Lung function, insulin resistance and incidence of cardiovascular disease: a longitudinal cohort study. J Intern Med 2003;253:574-81.

31. Engström G, Gerhardsson de Verdier M, Dahlbäck M, et al. BP variability and cardiovascular autonomic function in relation to forced expiratory volume: a population-based study. Chest 2009;136:177-83

32. Brychta RJ, Shiavi R, Robertson D, et al. A simplified two-component model of blood pressure fluctuation. Am J Physiol Heart Circ Physiol 2007;292:H1193-203.

33. Mancia G, Bombelli M, Facchetti R, et al. Long-term prognostic value of blood pressure variability in the general population: results of the Pressioni Arteriose Monitorate e Loro Associazioni Study. Hypertension 2007:49:1265-70.

34. Suzuki T, Katz R, Jenny NS, et al. Metabolic syndrome, inflammation, and the incident heart failure in the elderly: the cardiovascular health study. Circ Heart Fail 2008; 1:242-8.

35. Vasan RS, Sullivan LM, Roubenoff R, et al. Framingham Heart Study. Inflammatory markers and risk of heart failure in elderly subjects without prior myocardial infarction: the framingham heart study. Circulation 2003;107:1486-91. 